\title{
Foreword
}

\section{Seeing the world through a new set of glasses: Emerging technologies for the study of cell nuclei and chromosomes}

Since the earliest microscopes were used to examine cells, scientists have been intrigued by the long rodlike structures that appear during cell division. We now know that these structures are chromosomes and that they carry the full complement of a cell's genetic material. However, in many ways we are still at the beginning stages of understanding how these complex and intriguing structures work. How are the many parts of these genetic machines put together? Where do the key biological activities occur? How are these activities facilitated by the complex threedimensional (3D) structures of chromosomes found within the cell?

Answering these questions requires information from a variety of perspectives, from the molecular composition of chromosomes (DNA, histones, etc.) to detailed maps of these molecules once assembled into 3D chromosomes within the cell. On top of all that, chromosomes are dynamic structures, changing dramatically between mitosis and interphase and more subtly within these stages of the cell cycle. Thus, the mechanical and physical properties that permit the movement and rearrangement of chromosomes also are key to understanding how chromosomes function in reading out and faithfully replicating the genome.

The past decade has provided a wealth of information regarding the molecular composition of chromosomes, but relatively little is known about the structural, dynamic, and mechanical properties of these enormous macromolecular assemblies. These areas of investigation had lagged behind owing to seemingly insurmountable technical challenges. These challenges included the spatial resolution limits of light microscopy, the temporal resolution of live cell imaging, and the ability to detect and identify individual molecules within the cell. In addition, the capacity to measure a chromosome's mechanical properties was limited in a number of ways, including the ability to access chromatin within the cell. Fortunately, recent advances in a variety of technologies have broken these barriers on several fronts and have spurred a renaissance in chromosome structure studies. This special issue therefore highlights some of those new technologies, which hold great promise to unlock hidden secrets of chromosome structure and function.

The articles presented here encompass topics from the molecular composition of chromosomes to the topography and folding of the chromatin fiber, the dynamics of those structures, their connection with other parts of the nucleus, and the mechanics behind these interactions and folding states.

At the molecular level, chromosomes are incredibly complex. Figuring out how all the parts are put together represents an enormous challenge in and of itself. Whole-genome sequencing efforts provided essential information on the DNA backbone of each chromosome, but much work remains on how that DNA is assembled with hundreds of other molecules in vivo. A particularly important challenge is that the details of chromosome 3D structure are at macromolecular size scales, which are below the resolution of conventional light microscopy, the preferred method for 3D investigations. In answer to this challenge, Reymann et al. and Carlton describe recent developments in light microscopy that access details in the nucleus not observable by conventional techniques. Using two different types of structured illumination techniques, these new light microscopies reveal, for example, new topographical features of meiotic chromosomes and interphase chromatin domains in living nuclei. As a complementary method to light microscopy, Ushiki and Hoshi illustrate how atomic force microscopy (AFM) detects fine-scale topographical features of mitotic chromosomes in an aqueous environment.

High resolution has been the rule for electron microscopy (EM) for decades. EM has been applied to many key studies of chromosomes in mitotic and meiotic cells, where individual chromosomes are readily identifiable. However, probing chromosome 
structure in interphase nuclei by EM has been somewhat limited owing to the dense packing of this organelle. To overcome this problem, Bazett-Jones and colleagues have applied spectroscopic methods to EM, enabling molecular resolution of chromatin and other structures within the nucleus. In addition, Branco and Pombo discuss how EM samples can be prepared to label specific chromosomal sequences by in situ hybridization and yet still maintain nuclear ultrastructure. These technical feats promise to unlock the door to the spatial relationships between distinct molecules, nuclear 'compartments', and specific parts of the chromosome at unprecedented resolution.

The structure of a chromosome is constantly changing, as are its interactions with its environment (e.g. with mitotic spindle, nuclear bodies). Understanding chromosome dynamics and the transient structure-function relationships of chromosomes is important to fully comprehend chromosome activity. Live cell imaging has revealed that, even within interphase, chromatin is dynamic. Two powerful techniques that elegantly give access to dynamic parameters extract that information by looking at large populations of fluorescent molecules. Fluorescence recovery after photobleaching (FRAP) measures the recovery of fluorescence in a spot after a strong bleaching pulse. Fluorescence correlation spectroscopy (FCS) observes the fluctuations of the fluorescence signal of single molecules moving in and out of a similar spot. Renz and Langowski demonstrate the application of both of these techniques to explore the dynamics of the actin-binding protein CapG in the nucleus.

For cases such as single chromosomal loci, where ensemble behavior cannot be studied, or when single events are the focus of a study, single-particle or single-molecule techniques applied to chromatin have become more and more feasible. Yet the 3D nature of chromatin movement complicates tracking of single loci. Levi and Gratton present a new imaging technique that overcomes these limitations, enabling new insights into the 3D dynamics of fluorescently tagged loci or other nuclear entities. To analyze dynamic interactions on a molecular size scale, fluorescence (or Förster) resonance energy transfer (FRET) was developed several decades ago and has been applied to a range of biological questions. Recent technological advances now allow the observation of
FRET on the single-molecule level, revealing details hidden before in the ensemble of probe molecules. Leuba et al. describe in this issue several applications of FRET to investigate dynamic protein-DNA interactions on the single-molecule level.

The packing of a genome into a nucleus is an amazing feat of engineering. In mammalian cells, two meters of genomic DNA are folded and packed into nuclei that are only $\sim 10 \mu \mathrm{m}$ in diameter. The DNA from each chromosome is condensed 300- to 1000-fold and transitions between folding states throughout the life cycle of the cell. What are the mechanical forces that hold these complex and compact structures together and yet permit their movement, folding, and unfolding? The most information we have on the physical-mechanical properties of chromosomes comes from work with mitotic chromosomes. The article by Marko summarizes these studies as well as key biochemical features of chromatin, and then goes on to discuss new insights on the elastic properties of mitotic chromosomes using micropipette aspiration. From these diverse data, Marko proposes an intriguing model of chromosome folding, with a network of chromatin contacts cross-linked by non-DNA components that are not bound to each other. The physical properties of the chromatin network in interphase nuclei are discussed by Verstraeten and Lammerding, who describe the use of applied strain, micropipette aspiration, and AFM to determine the relative contributions of chromatin and the nuclear lamina to nuclear mechanics. In addition, Kanger et al. describe an experimental system that uses micro-magnetic tweezers to actively manipulate chromatin within living cells and probe its elastic properties.

Imaging and measuring of features of chromosome structure are not the end-points of a study. Critical to drawing accurate conclusions from data is the application of proper data analysis. Methods for data analysis are the focus of the article by Ronneberger et al. These authors highlight the application of the right measurements and statistics, as well as use of the right tools to make measurements in the first place. With an emphasis on the mainstay technique of fluorescence imaging, this article is especially useful to those just entering the field of chromosome research.

Since the first identification of chromosomes, advances in chromosome research have frequently 
been connected with new technological developments in imaging and data analysis techniques. We think that all methods presented in this issue have a practical relevance for chromosome research and provide new information, as can be seen from the applications shown in each article. Is this the last stage in the centuries-old quest to finally 'understand' nature at least in the cell nucleus? Certainly not. But new technological developments are already in the pipeline to allow even more insights into nature's secrets. Until then, the current collection represented by the articles in this issue offers us a large number of tools whose potential for chromosome research has not yet been exploited. Let's use them!

Lindsay Shopland \& Joerg Bewersdorf The Jackson Laboratory 600 Main Street Bar Harbor, ME 04609, USA

Lindsay.Shopland@jax.org Joerg.Bewersdorf@jax.org 